\title{
Development of a fully automated desktop chemical vapor deposition system for programmable and controlled carbon nanotube growth
}

\author{
Ju Hyun $\mathrm{Ahn}^{\dagger}$, Minho $\mathrm{Na}^{\dagger}$, Sungkwan Koo, Hyunsoo Chun, Inhwan Kim, Jong Won Hur, Jae Hyuk Lee
} and Jong G. OK (1)

\begin{abstract}
We have rationally designed and developed a fully automated desktop furnace system that enables programmable chemical vapour deposition growth of carbon nanotubes with controlled height, density, and pattern architecture. Comprising several essential components involving a heating furnace, mass flowmeters, and computer controller, the developed system realizes controlled and practical carbon nanotube growth without resort to expensive and ponderous instruments. By programming, modifying, and loading the reusable recipes in the developed system, systematic and reproducible growths of carbon nanotubes with desired morphology and dimension can be performed. Growth results with controlled height, density, and pattern are demonstrated through the actual operations, confirming the validity and usefulness of the developed system towards various practical applications.
\end{abstract}

Keywords: Carbon nanotube, Chemical vapor deposition, Furnace, Desktop system, Programmable growth recipe, Automated control, Micropattern, Nanopattern

\section{Introduction}

Since discovered almost two decades ago [1], carbon nanotubes (CNTs) have been extensively utilized in many diverse functional components and practical applications due to their extraordinary mechanical, electrical, chemical, and photonic characteristics [2-4]. To meet the unbounded needs in both academia and industry, reliable and rational manufacturing of CNTs with controlled density, desired length and diameter, and high quality has always been of great importance. Among several methods involving arc discharge and laser ablation, chemical vapour deposition (CVD) has played a central role in controlled CNT growth [5-8]. The CVDbased CNT growth method possesses many advantages

\footnotetext{
*Correspondence: jgok@seoultech.ac.kr

${ }^{\dagger} J u$ Hyun Ahn and Minho Na contributed equally to this work Department of Mechanical and Automotive Engineering, Seoul National University of Science and Technology, 232 Gongneung-ro, Nowon-gu, Seoul 01811, South Korea
}

compared to the other methods. It can generally provide highly crystalline, low-defect CNTs with good uniformity and reproducibility. It can lead to more scalable growth of CNTs well-bound to large area substrates, which can be more easily engineered, transferred, or harvested for subsequent uses. It also can readily control the density, alignment degree, length, and diameter of CNTs, based on the modulation of several CVD processing parameters such as gas composition and flow rate, temperature, and growth time. Additionally, CVD growth enables facile fabrication of CNT micro- and nanostructures by selective growth on the patterned catalyst.

However, the typical CVD system demands complex instrumentation and ponderous vacuum apparatus with numerous components most of which are not frequently used but costly. This restricts the otherwise much wider research and more practical development utilizing CNTs. As described in detail in our previous reports that our developed growth 'recipe' is based on the atmospheric 
pressure (AP) CVD [9], the CVD system for CNT growth does not necessitate complicated vacuum processing. Hence, what we essentially need may include the heating furnace, source gas feeder, and their controller. To this end, we develop a compact, desktop-scale CVD growth system comprising essential components with much reduced cost and volume, but effectively working for reliable and programmable CNT growth through the computer-controlled fully automated operation. In this work, we present the rational design and setup of the system, and demonstrate $\mathrm{CNT}$ growth results with controlled height, density, and alignment, as well as the specific micro- and nanopatterned architectures by operating the completed system with recipe modulations.

\section{Process overview and system requirement}

The APCVD process for CNT growth is briefly as follows. First, the catalyst layers comprising $\mathrm{Fe}$ on $\mathrm{Al}_{2} \mathrm{O}_{3}$ with nominal thicknesses of $1 \mathrm{~nm}$ and $10 \mathrm{~nm}$, respectively, are sequentially deposited on a $300 \mathrm{~nm}$-thick $\mathrm{SiO}_{2}$-coated $\mathrm{Si}$ wafer. Cut to the fit size, the catalyst pieces are loaded inside the CVD chamber at proper temperature gradient positions. After ample Ar purging, the chamber is heated up to the peak temperature $\left(\sim 800{ }^{\circ} \mathrm{C}\right)$ at a proper ramp rate under $\mathrm{Ar} / \mathrm{H}_{2}$ flowing. Dwelled at the peak temperature for catalyst annealing (mostly $\mathrm{H}_{2}$-driven reduction) for a while, $\mathrm{C}_{2} \mathrm{H}_{4}$ is then added as a carbon source for CNT growth. Once growth is done, the chamber is cooled down to room temperature under slight Ar flowing. Between runs, air baking is performed at around $800{ }^{\circ} \mathrm{C}$ for $30 \mathrm{~min}$ to clean the chamber by removing the inside carbon-based residues. The quantitative values of the gas flow composition, temperature ramp rate, peak temperature, time period for each step, and so on, can be tuned for optimizing the growth results, which will be discussed later.

In this regard, the CVD system may be designed to carry out the above-described process with precise controls of such parameters without excessive parts. Namely, the essential components of the system may consist of the heating chamber, four mass flow controller (MFC) channels designated for $\mathrm{Ar}, \mathrm{H}_{2}, \mathrm{C}_{2} \mathrm{H}_{4}$, and air, and the computer controller, along with surrounding plumbing and wiring.

\section{System design and setup: hardware and software}

Figure 1 illustrates the overall system design that can perform the necessary processes under computer control. First, we employed the $1^{\prime \prime}$-diameter single-zone tube furnace that can heat up to $1200{ }^{\circ} \mathrm{C}$ equipped with RS-232/485 serial communication ports (TF55035C-1, Lindberg/Blue $\mathrm{M}^{\mathrm{TM}}$ Mini-Mite ${ }^{\mathrm{TM}}$, Thermo Fisher Scientific, Ltd.), to use as a computer-controllable heating

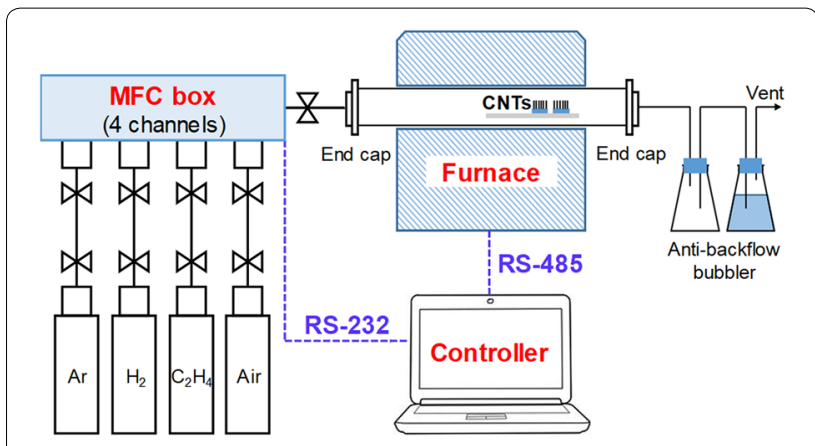

Fig. 1 Schematic diagram of the computer-controlled fully automated compact CVD system for CNT growth, consisting of the furnace, 4-channel MFC box, and controller. Serial communications between hardware components enable reliable and reproducible operation. Valves and anti-backflow bubbler ensure safety

chamber (Fig. 2a). An 1"-diamter quartz tube was assembled with the $\mathrm{O}$ ring-sealed stainless steel endcaps having the plumbing holes, and was mounted inside the tube furnace. Next, we designed and setup the MFC box (Fig. 2b; Sci-En Tech Corp.) comprising four MFC channels (AFC 500/600 series, ATOVAC Co., Ltd.) and their integrated throttle valve controller with a display monitor (GMC 1200, ATOVAC Co., Ltd.). Four MFCs were respectively connected to the designated gas tanks (as depicted in Fig. 1) through the stainless steel pipes and safety/regulator valves. The MFC box outlet was made by merging individual MFC outlets. Here to avoid dangerous flowing of $\mathrm{H}_{2}$ and air at the same time, a three-way valve was connected between $\mathrm{H}_{2}$ and air channels; either $\mathrm{H}_{2}$ or air could flow at a certain time, not ever simultaneously. The chamber inlet endcap hole and MFC box outlet valve were connected to each other by using a flexible stainless steel hose (Swagelok Company). The chamber outlet endcap hole was connected to the vent through the glycerine-filled bubbler that can prevent possible backflow, by using flexible Tygon ${ }^{\circledR}$ tubes (Saint Gobain Corp.). Finally, the furnace and MFC valve controller were wired to the computer via RS-485 and RS-232 communication cables, respectively.

The controlling software was designed and developed by using LabVIEW (version 2017, National Instruments Corp.), for the automated and reliable operations of the furnace and MFCs. Step-wise settings of temperatures, gas flow rates, and step period times can be put in the left side of the software's front panel shown in Fig. 2c, which transmit to and operate the furnace and MFC box via serial communications. The furnace temperature and gas flow rates can be real-time tracked in the right side monitor with the elapsed time as an $\mathrm{x}$-axis. The sensors installed in the furnace and MFC box, in conjunction with the LabVIEW monitor enable the feedback control 

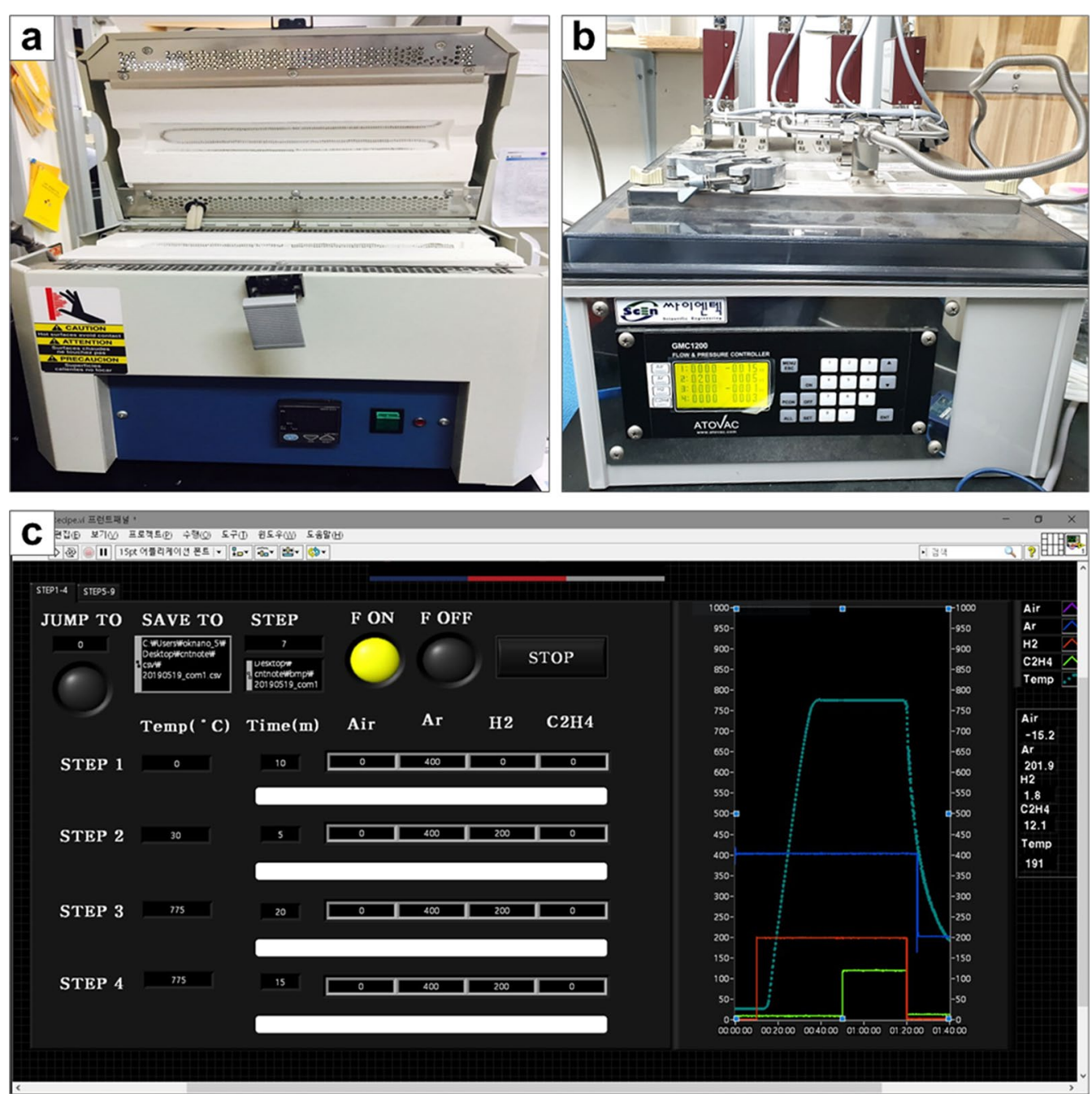

Fig. 2 Photographs of the main hardware and software components comprising the desktop CVD system for controlled CNT growths: a 1"-diameter tube furnace equipped with a serial-communicable temperature control module, b completed MFC box assembly containing four MFC channels (upper part) and their throttle valve controller (lower part), and c screenshot of the front panel of a CVD system-controlling LabVIEW software. The left side shows step-wise setting lines for temperatures, gas flows, and step period times, and the right side displays the real-time traces of such parameters during the process operation

of temperature and gas flows to maintain the set values with minimal disturbances. Temperature ramp and dwell can also be straightforwardly programmed in this software panel; if the temperature different from the previous step is set, the furnace ramps up or down during the set period time; if the same temperature is set as the previous step, the furnace temperature dwells during the set period time. Further, we added the "preset" function; the set values of temperatures, gas flow rates, and step period times for all step lines can be saved as an ASCII text file, and this file can be reloaded later for repeated uses (as a "recipe"). This "Export and Import" function can facilitate the recipe development and management for various CNT growth conditions.

\section{System operation results: controlled carbon nanotube growth}

As aforementioned, the system design and setup have been implemented with a focus on the compact volume and affordable cost. Figure $3 \mathrm{a}$ shows the completed CVD system. The system volume was cut down to $\sim 15 \%$ of the commercial CVD system (comparatively shown in Fig. 3b), and indeed could be put on the desktop with a minimal space. Meanwhile, the total expense was $~ 78 \%$ saved (from USD 55,000 to 12,500, approximately). While such a successful reduction in both volume and cost is mostly attributed to the curtail of vacuum-related and other uncritical components so far, we now evaluate the key function of the system, that is 

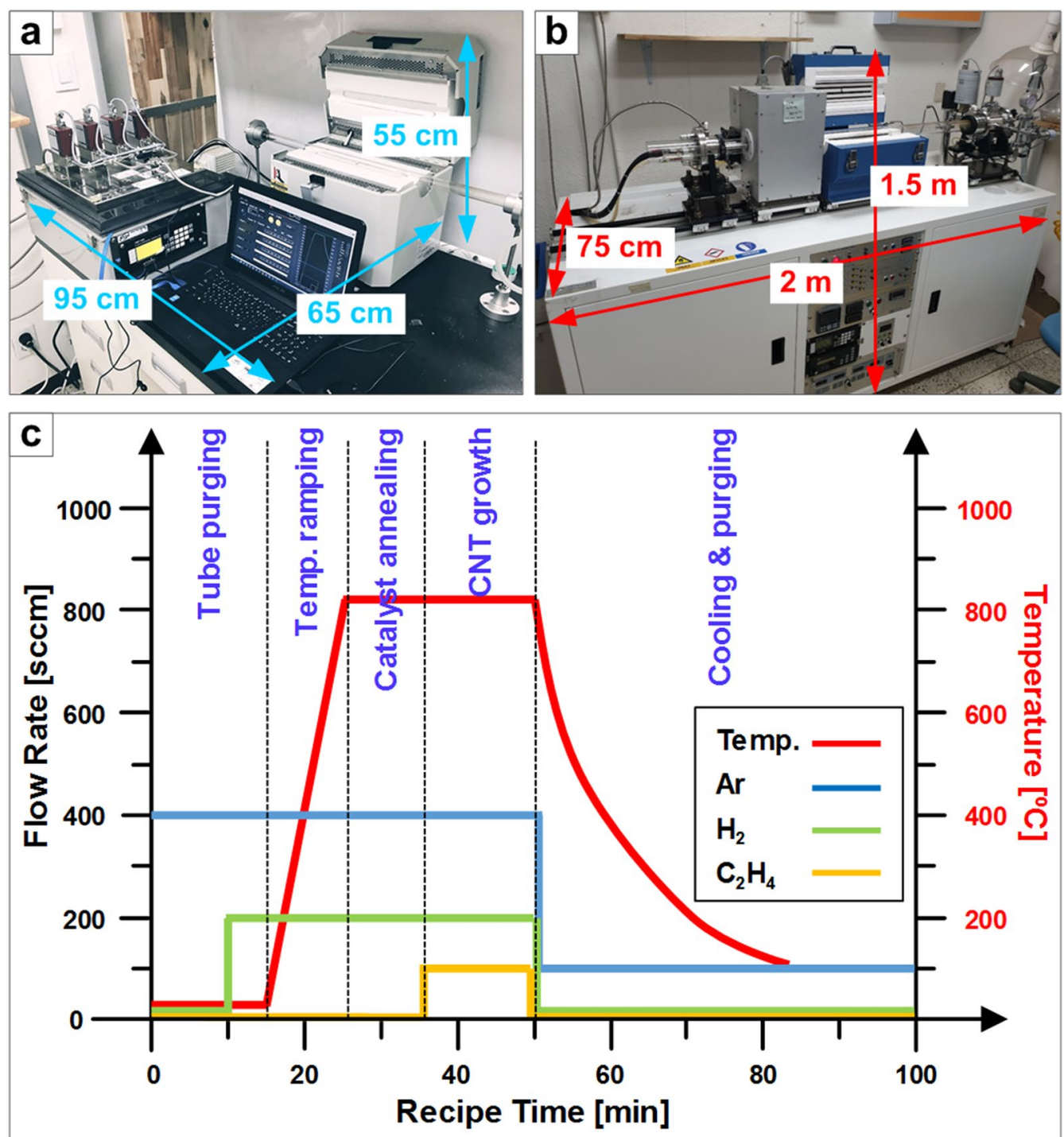

Fig. 3 Completed CVD system in comparison to a commercially-available one and the developed standard recipe: $\mathbf{a}$ our developed desktop CVD, $\mathbf{b}$ commercial CVD, and c standard recipe for CNT growth in the completed CVD system

programmable, reliable, and practical CNT growth, by actual operations.

Figure $3 \mathrm{c}$ demonstrates one of the recipes we have investigated for optimal CNT growth using the developed compact CVD system, where the prime parameters involving temperature, gas flow composition and rates, and times for each step for CNT growth are fully marked in detail. These recipes can be loaded, modified, saved, and reloaded in the LabVIEW controller for repeated controlled growths. Figure 4 shows the exemplary growth results obtained by using the recipe shown in Fig. 3c. The high-density CNTs (i.e., CNT "forest") were uniformly grown on the catalyst substrates with up to a millimetrescale height (Fig. 4a) and/or over the large area (Fig. 4b), confirming reliable and effective CNT growth. Figure 4c reveals typical nanoscale morphology of the as-grown CNT forests, with the average diameter and interspacing range of $10 \mathrm{~nm}$ and $50-100 \mathrm{~nm}$, respectively. The growth height can be controlled mainly by the growth time (time of $\mathrm{C}_{2} \mathrm{H}_{4}$ flowing, as shown in Fig. 3c); Fig. $4 \mathrm{~d}-\mathrm{f}$ disclose three different examples with different heights, obtained by different growth times.

Controlled growth of CNTs having specific density along with various alignment degree can also be readily conducted by modulation of recipe parameters. The density control can be induced by many means such as feed gas regulation $[10,11]$ and catalyst patterning [12-14], but here we demonstrate one strategy for density control 

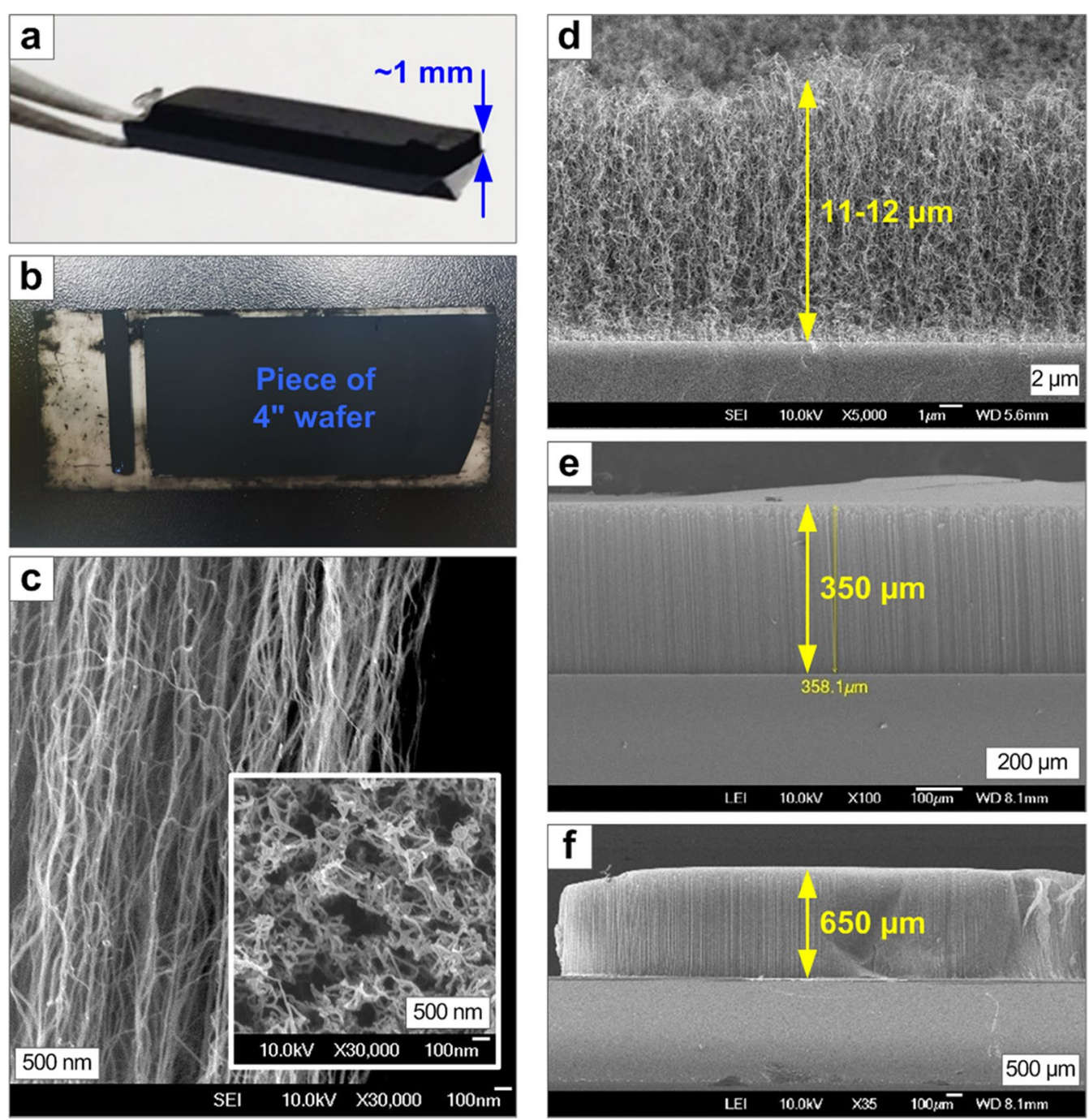

Fig. 4 CNT forests grown in the developed CVD system: $\mathbf{a}$ and $\mathbf{b}$ photographs of exemplary samples, $\mathbf{c}$ nanoscale morphology of the sidewalls of as-grown CNTs (inset shows the top view), $\mathbf{d}-\mathbf{f}$ CNT forests with different heights controlled by growth time

based on the modulation of annealing time (i.e., time of $\mathrm{Ar} / \mathrm{H}_{2}$ flowing at the peak temperature, as marked in Fig. 3c) [15]. Figure 5 shows three cases of CNTs having different areal densities grown by varying the annealing time; annealing times were controlled to $5 \mathrm{~min}, 10 \mathrm{~min}$, and $20 \mathrm{~min}$ for cases 1,2 , and 3, respectively. Insets to the left-side top images in Fig. 5, taken from the 'blank' runs (i.e., recipe runs with no $\mathrm{C}_{2} \mathrm{H}_{4}$ flowing), reveal the morphologies of annealed catalysts for each case. From the right-side sidewall images of Fig. 5, we can find that CNTs are better aligned with increased density at shorter annealing time in our experimental space; even shorter annealing time may end up with insufficient catalyst annealing. During the annealing step, the thin Fe catalyst film is clustered to nanoscale particles on the $\mathrm{Al}_{2} \mathrm{O}_{3}$ layer. The longer the annealing time is (case 3), the $\mathrm{Fe}$ nanocluster size becomes larger while the number of clusters decreases as agglomerated together [15]. This leads to low-density, vertically less-aligned CNTs. On the other hand, when grown from the catalyst nanoclusters with higher density (case 1), the resulting CNT density becomes higher [16]. In this case, CNTs may grow more upright, as the interspaces between neighbouring CNTs get smaller (i.e., "crowding effect") [17].

Finally, we demonstrate that the system enables the direct growth of CNT architectures comprising specifically designed micro- and nanoscale patterns, through selective CNT growth on patterned catalysts. The catalyst layers can be patterned by suitable methods such as photolithography [18] and nanoimprint lithography [13]. The recipe modification can be performed to optimize the selective growth of CNT patterns, 

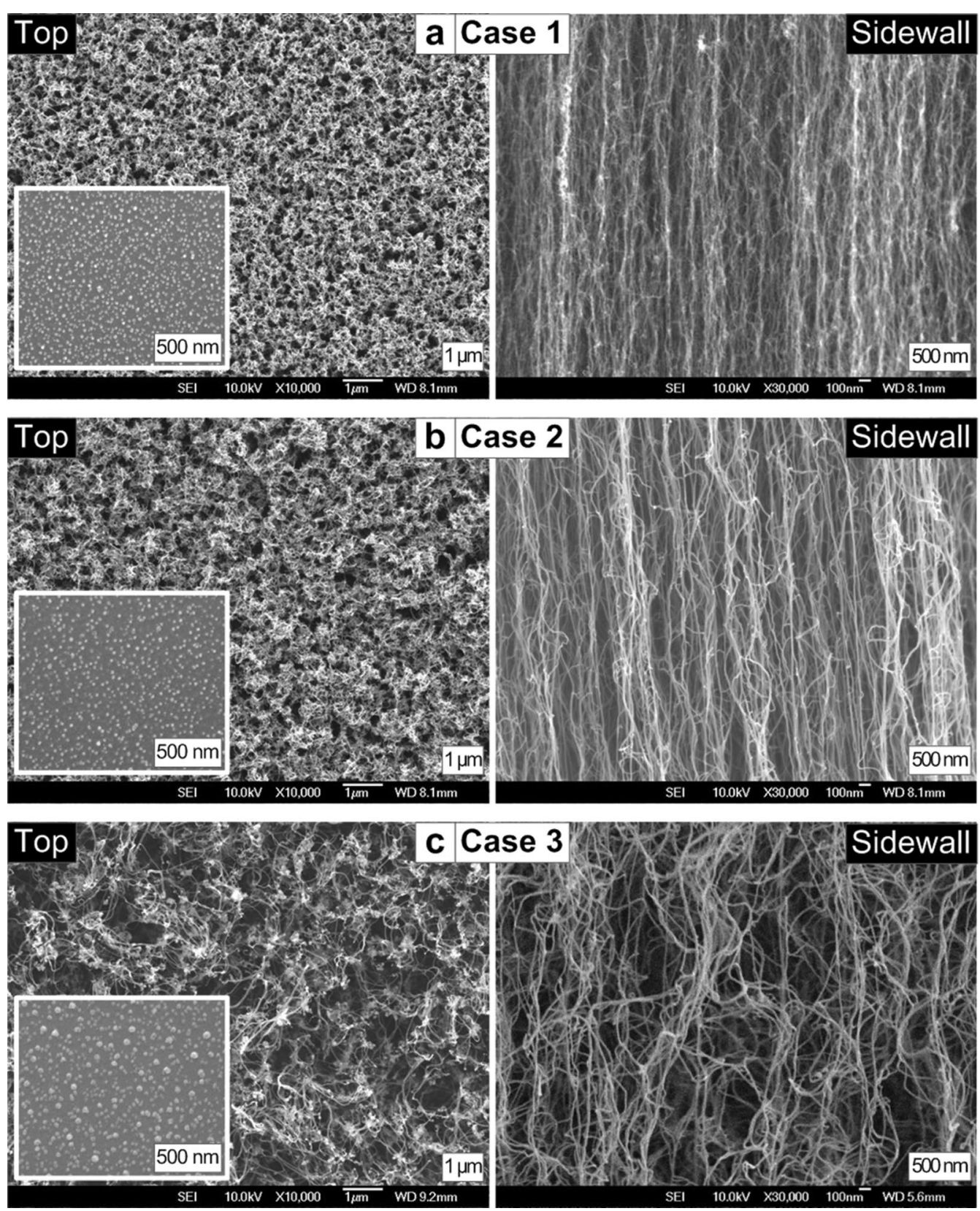

Fig. 5 Three cases of CNTs having different areal densities and alignments grown by varying annealing times: The annealing times were set for a $5 \mathrm{~min}, \mathbf{b} 10 \mathrm{~min}$, and $\mathbf{c} 20 \mathrm{~min}$. Left and right images show the top and sidewall SEM images of each case, respectively. Insets show the catalyst morphologies taken from blank runs for each case

usually by controls of annealing time and $\mathrm{C}_{2} \mathrm{H}_{4}$ flow rate. Such micro- and nanoarchitectures with specifically designed CNT structures can be capitalized in many devices involving mechanics, photonics, metastructures, sensors, and energy converters [3, 19], as well as provide the templates for growing other complimentary nanomaterials towards more functional hybrid architectures [9, 18]. While further investigation on the systematic fabrications of diverse application-specific CNT architectures is underway for the following report, Fig. 6 shows several examples of the patterned CNT growths, confirming that the developed CVD system achieves reliable and practical CNT architecture fabrication. 

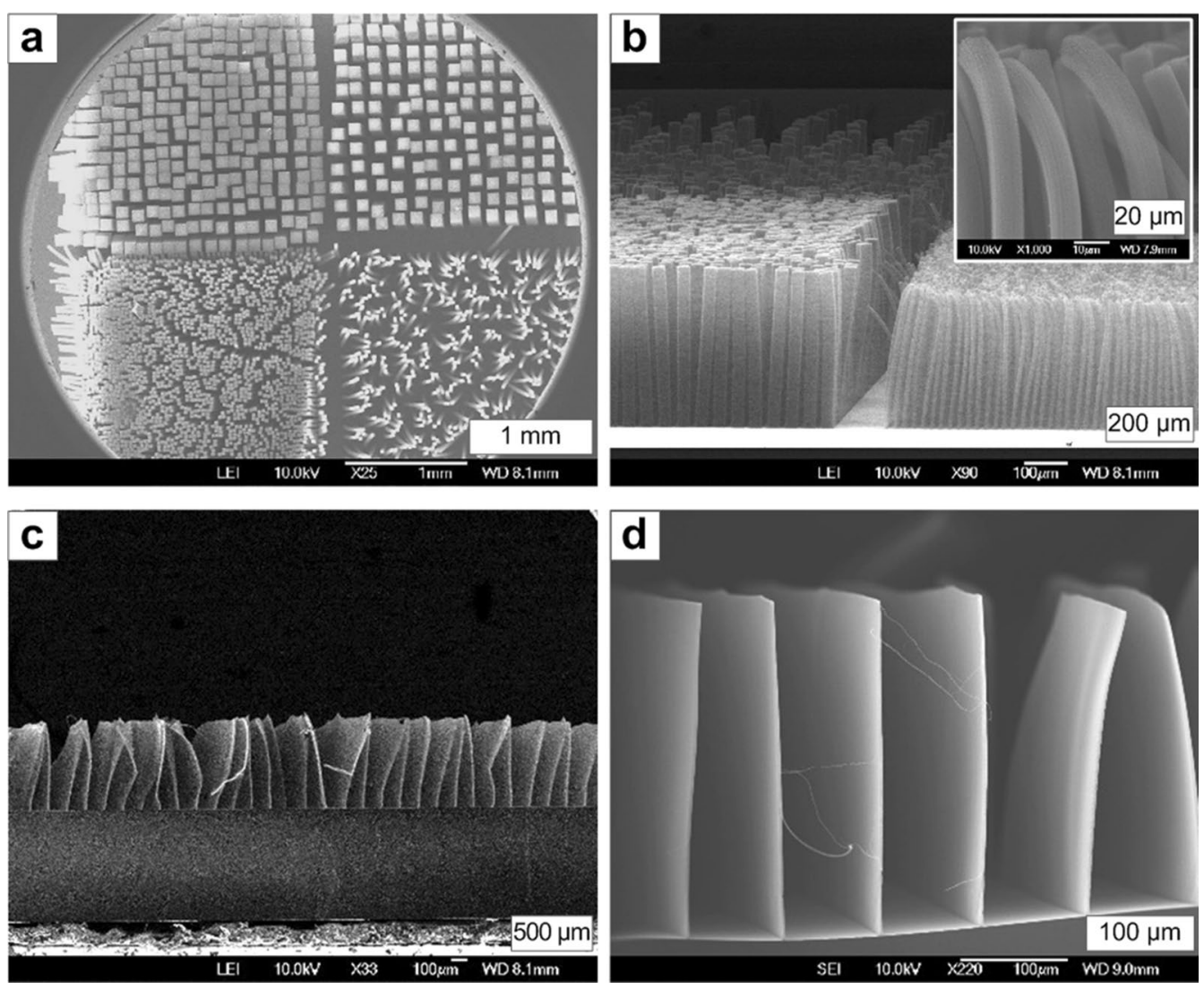

Fig. 6 Fabrication examples of CNT architectures comprising micro- and nanoscale patterns by using the developed CVD system: a and $\mathbf{b}$ CNT micropillar arrays with various diameters and $\mathbf{c}$ and $\mathbf{d}$ microsheet patterns

\section{Conclusions}

In summary, we have developed a computer-controlled compact CVD system that enables programmable growths of CNTs with desired height, density, and pattern architecture. Rational system design and setup of essential components involving a heating furnace, MFCs, and computer controller, realize the fully automated desktop-scale system with significantly reduced volume and cost, compared to commercial CVD instruments. We have demonstrated that the controlled CNT growth with desired morphology and dimension could be conducted by programming, modifying, and loading the reusable recipes in the developed system. This study will facilitate practical developments of many applications utilizing CNTs and their derivative architectures such as CNT thin films, coatings, and micro- and nanoarchitectures.

\section{Acknowledgements}

We thank Bo Kyung Kim for assistance with SEM imaging and the National NanoFab Center (NNFC) for assistance with microfabrication.

\section{Authors' contributions}

JGO conceived, designed, and supervised the research. All authors contributed to experiments, characterizations, data analyses, and manuscript preparation. All authors read and approved the final manuscript.

\section{Funding}

This work was supported by the Research Program funded by the Seoul National University of Science and Technology (Grant Number 2018-0788)

\section{Availability of data and materials}

All data generated or analysed during this study are included in the article.

\section{Competing interests}

The authors declare that they have no competing interests.

Received: 30 May 2019 Accepted: 22 Auqust 2019

Published online: 26 August 2019

\section{References}

1. lijima S (1991) Helical microtubules of graphitic carbon. Nature 354:56-58

2. Vairavapandian D, Vichchulada P, Lay MD (2008) Preparation and modification of carbon nanotubes: review of recent advances and applications in catalysis and sensing. Anal Chim Acta 626:119-129

3. De Volder MFL, Tawfick SH, Baughman RH, Hart AJ (2013) Carbon nanotubes: present and future commercial applications. Science 339:535-539

4. Shah KA, Tali BA (2016) Synthesis of carbon nanotubes by catalytic chemical vapour deposition: a review on carbon sources, catalysts and substrates. Mater Sci Semicond Process 41:67-82

5. Kumar M, Ando Y (2010) Chemical vapor deposition of carbon nanotubes: a review on growth mechanism and mass production. J Nanosci Nanotechnol 10:3739-3758

6. Handuja S, Srivastava P, Vankar VD (2010) On the growth and microstructure of carbon nanotubes grown by thermal chemical vapor deposition. Nanoscale Res Lett 5:1211-1216 
7. Ghoranneviss M, Elahi AS (2016) Review of carbon nanotubes production by thermal chemical vapor deposition technique. Mol Cryst Liq Cryst 629:158-164

8. See $\mathrm{CH}$, Harris AT (2007) A review of carbon nanotube synthesis via fluidized-bed chemical vapor deposition. Ind Eng Chem Res 46:997-1012

9. Ok JG, Tawfick SH, Juggernauth KA, Sun K, Zhang YY, Hart AJ (2010) Electrically addressable hybrid architectures of zinc oxide nanowires grown on aligned carbon nanotubes. Adv Func Mater 20:2470-2480

10. Kunadian I, Andrews R, Qian DL, Menguc MP (2009) Growth kinetics of MWCNTs synthesized by a continuous-feed CVD method. Carbon 47:384-395

11. Jung D, Han M, Lee GS (2014) Regrowth analysis of multiwalled carbon nanotube forests. Appl Phys Express 7:4

12. Gangele A, Sharma CS, Pandey AK (2017) Synthesis of patterned vertically aligned carbon nanotubes by PECVD using different growth techniques: a review. J Nanosci Nanotechnol 17:2256-2273

13. Park SJ, Ok JG, Park HJ, Lee KT, Lee JH, Kim JD, Cho E, Baac HW, Kang S, Guo L, Hart AJ (2018) Modulation of the effective density and refractive index of carbon nanotube forests via nanoimprint lithography. Carbon 129:8-14

14. Razib MAB, Saleh $T$ (2019) A review on micro-patterning processes of vertically aligned carbon nanotubes array (VACNTs array). Curr Nanosci 15:328-353
15. Bedewy M, Meshot ER, Guo HC, Verploegen EA, Lu W, Hart AJ (2009) Collective mechanism for the evolution and self-termination of vertically aligned carbon nanotube growth. J Phys Chem C 113:20576-20582

16. Bedewy M, Meshot ER, Reinker MJ, Hart AJ (2011) Population growth dynamics of carbon nanotubes. ACS Nano 5:8974-8989

17. Meshot ER, Bedewy M, Lyons KM, Woll AR, Juggernauth KA, Tawfick S, Hart AJ (2010) Measuring the lengthening kinetics of aligned nanostructures by spatiotemporal correlation of height and orientation. Nanoscale 2:896-900

18. Ok JG, Lee JY, Baac HW, Tawfick SH, Guo LJ, Hart AJ (2014) Rapid anisotropic photoconductive response of $\mathrm{ZnO}$-coated aligned carbon nanotube sheets. ACS Appl Mater Interfaces 6:874-881

19. Tawfick S, De Volder M, Copic D, Park SJ, Oliver CR, Polsen ES, Roberts MJ, Hart AJ (2012) Engineering of micro- and nanostructured surfaces with anisotropic geometries and properties. Adv Mater 24:1628-1674

\section{Publisher's Note}

Springer Nature remains neutral with regard to jurisdictional claims in published maps and institutional affiliations.

\section{Submit your manuscript to a SpringerOpen ${ }^{\circ}$ journal and benefit from:}

- Convenient online submission

- Rigorous peer review

- Open access: articles freely available online

- High visibility within the field

- Retaining the copyright to your article

Submit your next manuscript at $\boldsymbol{\nabla}$ springeropen.com 